\title{
Congratulations to Arnold Demain
}

\author{
Alexander Steinbüchel ${ }^{1}$
}

Published online: 20 March 2017

(C) Springer-Verlag Berlin Heidelberg 2017

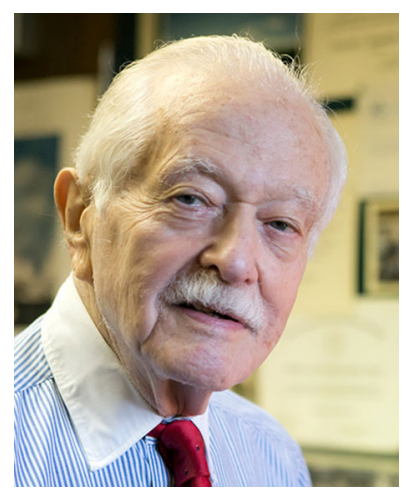

On April 26, Professor Arnold Demain will celebrate his 90th birthday, and we wish to congratulate him on this milestone. Arnold Demain is an Associate Editor of 'Applied Microbiology and Biotechnology', and was there when the journal was first founded in 1975-he has now connected to the journal for four decades!

Arnold Demain was born in Brooklyn in 1927. Likely inspired by his father and uncles, who were successfully operating pickles factories, he received first training as a food fermentation expert at Michigan State University in East Lansing. His training was interrupted by military service at the end of World War II, before he returned to the same uni-

Alexander Steinbüchel

steinbu@uni-muenster.de

Institut für Molekulare Mikrobiologie und Biotechnologie, Westfälische Wilhelms-Universität Münster, Corrensstraße 3, 48149 Münster, Germany versity to obtain his Bachelor's and then his Master's degree in 1949 and 1950, respectively. Afterwards, he transferred to the University of California at Berkeley and at Davis, where he received his Ph.D. in 1954 after having examined pectolytic enzymes. In the same year, he started a job at the company 'Merck Sharp \& Dohme,' where he soon became responsible for the production of penicillin.

In 1964, he became the head of a new department aiming at the improvement of microbial strains for the production of various kinds of biotechnological products. In 1969, he left Merck to become a Professor of Industrial Microbiology at Massachusetts Institute of Technology (MIT). In 1971, he became intensively engaged with Cetus Corporation in Berkeley, the first biotechnology company founded in the USA. Since becoming an Emeritus Research Fellow at Drew University in New Jersey in 2001, he has worked to pass on his knowledge to undergraduate students and to 'inoculate' them for their future careers.

In addition to receiving five honorary doctorates (Universities of Leon, Ghent, Technion, Michigan State, and Münster), Arnold Demain is also a member of several National Academies of Sciences (USA, Mexico, Hungary) and serves on the Board of Governors of the American Academy of Microbiology. His expertise has made him one of the world's most eminent industrial microbiologists. As a result, he has also had an invaluable impact for the journal's very successful development, and it has always been very inspiring and stimulating to meet him personally or during Editorial Board meetings for the journal. I am very grateful for these talks, as well as his valued and tireless support, and would like to wish him the very best for the future!

Münster, in April 2017Alexander Steinbüchel 